\title{
LARGE-TIME BEHAVIOR OF SOLUTIONS OF LAX-FRIEDRICHS FINITE DIFFERENCE EQUATIONS FOR HYPERBOLIC SYSTEMS OF CONSERVATION LAWS
}

\author{
I-LIANG CHERN
}

\begin{abstract}
We study the large-time behavior of discrete solutions of the LaxFriedrichs finite difference equations for hyperbolic systems of conservation laws. The initial data considered here are small and tend to a constant state at $x= \pm \infty$. We show that the solutions tend to the discrete diffusion waves at the rate $O\left(t^{-3 / 4+1 / 2 p+\sigma}\right)$ in $l^{p}, 1 \leq p \leq \infty$, with $\sigma>0$ being an arbitrarily small constant. The discrete diffusion waves can be constructed from the self-similar solutions of the heat equation and the Burgers equation through an averaging process.
\end{abstract}

\section{INTRODUCTION}

In numerical calculations of a shock wave in gas flows, one usually observes some noise emitted from the shock wave. At large time, noise usually forms certain shapes and moves at constant speeds. When one uses first-order finite difference methods, this noise looks like humps and spreads out, but the $l^{1}$ norms do not decay. If one uses second-order methods, the noise appears as wiggles or solitary waves. In dealing with hyperbolic systems of conservation laws, one encounters noise not only in the calculation of a shock wave, but also in the calculation of a rarefaction wave, or even in a flow near a constant state. The noise patterns, or error waves, move at constant characteristic speed in large time and decay slowly. They are a primary source of error and may ruin the downstream flow pattern. It is therefore important to understand their formation and propagation. Furthermore, understanding these error waves is a key step in the study of stability of discrete entropy shock waves and discrete rarefaction waves for a finite difference scheme. It is also a key step in understanding the persistence of a nonentropy shock in some second-order finite difference schemes.

In this paper, we study the error waves of the Lax-Friedrichs scheme for general hyperbolic systems of conservation laws. We characterize these error

Received May 19, 1989; revised February 15, 1990.

1980 Mathematics Subject Classification (1985 Revision). Primary 39A12; Secondary 35L65.

Key words and phrases. Lax-Friedrichs scheme, hyperbolic systems of conservation laws, discrete diffusion waves, asymptotic behavior, numerical viscosity. 
waves as discrete diffusion waves. In the simplest case, these waves are the largetime asymptote of solutions of the Lax-Friedrichs finite difference equations, with initial data being a perturbation of a constant state. Thus, we wish to study the large-time behavior of solutions of the Lax-Friedrichs finite difference equations

$$
\begin{aligned}
U(j, n)= & \frac{U(j-1, n-1)+U(j+1, n-1)}{2} \\
& +\frac{\lambda}{2}(f(U(j-1, n-1))-f(U(j+1, n-1))), \\
& \quad U \in R^{N}, n>0, j+n=\text { even, }
\end{aligned}
$$

subject to the condition on the initial data,

$$
U(j, 0) \rightarrow \text { constant as }|j| \rightarrow \infty .
$$

Without loss of generality, we may take this constant state to be the zero state:

$$
U(j, 0) \rightarrow 0 \text { as }|j| \rightarrow \infty .
$$

System (1.1) is the Lax-Friedrichs discretization of the following system:

$$
u_{t}+f(u)_{x}=0, \quad u \in R^{N},-\infty<x<\infty, t>0,
$$

with $\lambda$ being $\Delta t / \Delta x . \Delta t$ and $\Delta x$ are, respectively, the temporal and spatial mesh widths of the scheme. System (1.3) is assumed to be strictly hyperbolic, which means that $f^{\prime}(u)$ has real and distinct eigenvalues $\lambda_{1}(u)<\cdots<\lambda_{N}(u)$ with right eigenvectors $r_{i}(u)$ and left eigenvectors $l_{i}(u), i=1, \ldots, N$. In (1.1), $\lambda>0$ and is required to satisfy the following strict Courant-FriedrichsLewy condition:

$$
\min _{i, u}\left\{1-\left(\lambda \lambda_{i}(u)\right)^{2}\right\} \geq \nu>0 .
$$

Because of (1.4), the Lax-Friedrichs scheme introduces a positive amount of numerical viscosity. Its solution is expected to have a qualitative behavior similar to that of the solution of the following viscous conservation laws:

$$
u_{t}+f(u)_{x}=\left(B u_{x}\right)_{x}
$$

with initial data

$$
u(x, 0) \rightarrow 0 \text { as }|x| \rightarrow \infty .
$$

Here, $B$ is some viscosity matrix and satisfies certain parabolic conditions [5]. In this continuous system, the decay of solutions of (1.5) and (1.6) in $L^{2}$ and $L^{\infty}$ has been studied in $[5,8]$ (see references therein). The solutions do not decay in $L^{1}$ because the mass is conserved:

$$
\int_{-\infty}^{\infty} u(x, t) d x=\text { constant }
$$

Indeed, the solutions tend to a linear or nonlinear diffusion wave in each characteristic direction $[1,2,6]$. Each nonlinear (linear) diffusion wave is a self-similar solution of the Burgers (heat) equation. This is analogous to the N-waves in the 
system of hyperbolic conservation laws [7]. In this paper we show that, in the discrete case, the solutions of (1.1) and (1.2) decay in $l^{p}, 1<p \leq \infty$. Further, the $l^{1}$-asymptotes of the solutions consist of the discrete diffusion waves in each characteristic direction. They are analogous to those diffusion waves produced by the continuous viscous conservation laws. Indeed, discrete diffusion waves can be constructed from the continuous diffusion waves through an averaging process over the spatial grids, and they can be determined from the initial data a priori. Specifically, our main theorem, presented in $\S 3$, states that the solutions of (1.1) and (1.2) converge to these discrete diffusion waves at the rate $O(1+n)^{-1 / 4+\sigma}$ in $l^{1}$, with $\sigma>0$ being an arbitrarily small number.

Notation. The discrete functions considered here are defined over either odd or even integers. The $l^{p}$ space consists of all such functions with the finite $l^{p}$ norm

$$
\|U\|_{l^{p}}=\left(\sum|U(j)|^{p}\right)^{1 / p}<\infty .
$$

Here, the summation is over either odd or even integers depending on the domain of $U$. We define the following operators on the $l^{p}$ space: the translation operator $(T U)(j)=U(j+1)$, the average operator $A=\frac{1}{2}\left(T+T^{-1}\right)$, and the difference quotient $D=\frac{1}{2}\left(T-T^{-1}\right)$.

\section{DisCRETE DiffUSION WAVES}

Let us briefly explain the formation of the discrete diffusion waves of $(1.1)$ and (1.2). We abbreviate the discrete functions $U(\cdot, n), W(\cdot, n)$, etc., by $U(n), W(n)$, etc., and $\lambda_{i}(0), r_{i}(0), l_{i}(0)$ by $\lambda_{i}, r_{i}, l_{i}$, respectively. In (1.1), we decompose $U(n)$ into $\sum U_{i}(n) r_{i}$ and expand the flux function $f$ into a Taylor series about 0 up to second order. Then the $i$ th component of $(1.1)$ in the $r_{i}$ direction is

$$
U_{i}(n)=L_{i} U_{i}(n-1)-\lambda D\left(\sum_{j, k} \frac{b_{i j k}}{2} U_{j}(n-1) U_{k}(n-1)+H_{i}(U(n-1))\right) .
$$

Here, $L_{i}=A-\lambda \lambda_{i} D, b_{i j k}=l_{i} \cdot f^{\prime \prime}(0)\left(r_{j}, r_{k}\right)$, and $H_{i}$ represents the higherorder terms of $f$. Roughly speaking, the formation of the discrete diffusion waves is due to two factors. First, mass is conserved; i.e., $\sum U_{i}(j, n)=m_{i}$ $\forall n \geq 0$. Second, system (1.1) becomes decoupled at large time, because of the strict hyperbolicity (this will be justified in the proof of our main theorem below). From this decoupling principle, one expects that at large time, in (2.1), the high-order terms $H_{i}$ and the transversal interaction terms $\sum_{j \neq k} b_{i j k} U_{j}(n-1)$. $U_{k}(n-1)$ become less important.

The primary nonlinear term in the $i$ th equation is $b_{i i i} U_{i}(n-1)^{2}$, and the second most important term is $\sum_{k \neq i} b_{i k k} U_{k}^{2}(n-1)$. One expects that the primary term of the solution $U_{i}(n)$ of $(2.1)$ should satisfy the following scalar 
equation:

$$
\begin{gathered}
\Theta_{i}(n)=L_{i} \Theta_{i}(n-1)-\lambda D\left(\frac{b_{i i i}}{2} \Theta_{i}^{2}(n-1)\right), \\
\Theta_{i}(j, 0)= \begin{cases}m_{i}, & j=0, \\
0, & \text { otherwise. }\end{cases}
\end{gathered}
$$

Thus, this primary wave $\Theta_{i}$ carries the conserved mass $m_{i}$.

The second most important term of the solution $U_{i}(n)$ should come from the following equation:

$$
\begin{gathered}
\Xi_{i}(n)=L_{i} \Xi_{i}(n-1)-\lambda D\left(\sum_{k \neq i} \frac{b_{i k k}}{2} \Theta_{k}^{2}(n-1)\right), \\
\Xi_{i}(j, 0)=0 .
\end{gathered}
$$

Our strategy is to write $U_{i}=\Theta_{i}+\Xi_{i}+W_{i}$ and then to estimate $\Theta_{i}$ and $\Xi_{i}$ and to show that $W_{i}$ is small at large time.

Notice that (2.2) and (2.3) of the primary wave $\Theta_{i}$ is the Lax-Friedrichs discretization of the inviscid Burgers equation. We wish to have an exact expression of $\Theta_{i}$ because it will be the large-time asymptote of the solutions of (1.1) and (1.2). Unfortunately, an exact expression is difficult to find. Nevertheless, an average over spatial grid cells of the continuous diffusion wave $\theta_{i}$, defined by

$$
\begin{gathered}
\theta_{i_{t}}+\lambda_{i} \theta_{i_{x}}+\frac{b_{i i i}}{2}\left(\theta_{i}^{2}\right)_{x}=\frac{1}{2} \frac{\nu_{i}}{\lambda} \theta_{i_{x x}}, \\
\theta_{i}(x, 0)=m_{i} \delta(x), \\
\nu_{i}=1-\left(\lambda \lambda_{i}\right)^{2},
\end{gathered}
$$

can serve as an approximate solution of (2.2) and (2.3) at large time. This continuous diffusion wave has the following exact expression [4]:

$$
\begin{gathered}
\theta_{i}(x, t)=\frac{1}{\sqrt{\left(t \nu_{i} / \lambda\right)}} \theta_{i}^{*}\left(\frac{x-\lambda_{i} t}{\sqrt{\left(t \nu_{i} / \lambda\right)}}\right), \\
\theta_{i}^{*}(\bar{x})=\left\{\begin{array}{lc}
\frac{m_{i}}{\sqrt{2 \pi}} e^{-\bar{x}^{2} / 2} & \text { if } b_{i i i}=0, \\
-\frac{\nu_{i}}{b_{i i i} \lambda}\left(\ln \psi_{i}(\bar{x})\right)^{\prime} & \text { if } b_{i i i} \neq 0,
\end{array}\right. \\
\psi_{i}(\bar{x}) \equiv e^{b_{m i} m_{t} / 2} \int_{-\infty}^{\bar{x}} e^{-y^{2} / 2} d y+e^{-b_{m} m_{t} / 2} \int_{\bar{x}}^{\infty} e^{-y^{2} / 2} d y .
\end{gathered}
$$

The ith discrete diffusion wave is then defined by

$$
\bar{\theta}_{i}(j, n) \equiv \frac{1}{2} \int_{-1}^{1} \theta_{i}(j+x,(n+1) \lambda) d x, \quad j+n=\text { even. }
$$


Clearly, $\sum_{j} \bar{\theta}_{i}(j, n)=m_{i} \forall n$. Thus, $\bar{\theta}_{i}$ carries the conserved mass $m_{i}$. Therefore, it does not decay in $l^{1}$. The following lemma justifies that $\bar{\theta}_{i}$ is a good approximation to $\Theta_{i}$ in large time.

Lemma 2.1. The discrete wave $\bar{\theta}_{i}$ has the following optimal estimates: For all $p$ with $1 \leq p \leq \infty$,

$$
\left\|\bar{\theta}_{i}(n)\right\|_{l^{p}}=O\left(m_{i}\right)\left(1+n \nu_{i}\right)^{-1 / 2+1 / 2 p} \text { as } n \rightarrow \infty .
$$

Furthermore, $\bar{\theta}_{i}$ satisfies

$$
\bar{\theta}_{i}(n)=L_{i} \bar{\theta}_{i}(n-1)-\lambda D\left(\frac{b_{i i i}}{2} \bar{\theta}_{i}^{2}(n-1)-E_{i}(n-1)\right),
$$

with the error $E_{i}$ having the following estimate: For all $p$ with $1 \leq p \leq \infty$,

$$
\left\|E_{i}(n)\right\|_{l^{p}}=O\left(m_{i}\right)\left(1+n \nu_{i}\right)^{-3 / 2+1 / 2 p} \text { as } n \rightarrow \infty .
$$

Proof. From (2.9), $\theta_{i}$ has the following optimal estimates: For all $p$ with $1 \leq p \leq \infty$, and for all $\alpha \geq 0$,

$$
\left(\int\left|\frac{\partial^{\alpha}}{\partial x^{\alpha}} \theta_{i}(x, t)\right|^{p} d x\right)^{1 / p}=O\left(m_{i}\right) O\left(\frac{\nu_{i}}{\lambda} t\right)^{-\alpha / 2-1 / 2+1 / 2 p} \text { as } t \rightarrow \infty
$$

Thus, (2.11) follows from (2.10) and (2.14). We now expand the function $\theta_{i}$ of (2.12) in a Taylor series about $\left(j-\lambda \lambda_{i}, n \lambda\right)$ and then use (2.6), (2.8), and (2.10) to obtain the expression of $E_{i}(j+1, n-1)$ as follows:

$$
\begin{aligned}
& E_{i}(j+1, n-1) \\
& =-\frac{b_{i i i}}{2}\left(2 \theta_{i}(j+1, n \lambda) \cdot K_{i}+K_{i}^{2}\right) \\
& \quad-\lambda \int_{0}^{1}(1-s)\left(\frac{b_{i i i}^{2}}{4} \theta_{i}^{3}-\frac{3}{4} \frac{b_{i i i} \nu_{i}}{\lambda} \theta_{i} \theta_{i_{x}}+\frac{\nu_{i}^{2}}{4 \lambda^{2}} \theta_{i_{x x x}}\right) \\
& \quad\left(j+1+(s-1) \lambda \lambda_{i},(n+s) \lambda\right) d s \\
& +\frac{\nu_{i}}{4 \lambda} \int_{0}^{1}(1-y)^{2}\left(\left(1+\lambda \lambda_{i}\right)^{2} \theta_{i_{x x}}\left(j+1+\lambda \lambda_{i}(y-1)+y, n \lambda\right)\right. \\
& \left.-\left(1-\lambda \lambda_{i}\right)^{2} \theta_{i_{x x}}\left(j+1+\lambda \lambda_{i}(y-1)-y, n \lambda\right)\right) d y,
\end{aligned}
$$

where

$$
K_{i}=\frac{1}{4} \int_{0}^{1}(1-y)^{2}\left(\theta_{i_{x x}}(j+1-y, n \lambda)+\theta_{i_{x x}}(j+1+y, n \lambda)\right) d y .
$$

Then (2.13) follows easily from (2.14)-(2.16).

This averaging process can also be applied to find an approximate solution for the wave $\Xi_{i}$ of (2.4)-(2.5). We consider the following equation:

$$
\xi_{i_{t}}+\lambda_{i} \xi_{i_{x}}+\left(\sum_{k \neq i} \frac{b_{i k k}}{2} \theta_{k}^{2}\right)_{x}=\frac{\nu_{i}}{2 \lambda} \xi_{i_{x x}}
$$




$$
\xi_{i}(x, 0)=0 .
$$

We define

$$
\bar{\xi}_{i}(j, n)=\frac{1}{2} \int_{-1}^{1} \xi_{i}(j+x,(n+1) \lambda) d x .
$$

Lemma 2.2. The discrete wave $\bar{\xi}_{i}$ has the following estimate: For all $p$ with $1 \leq p \leq \infty$, and $\sigma>0$ arbitrarily small,

$$
\left\|\bar{\xi}_{i}(n)\right\|_{l^{p}}=O\left(m_{i}^{2}\right)\left(1+n \nu_{i}\right)^{-3 / 4+1 / 2 p+\sigma} .
$$

Furthermore, $\bar{\xi}_{i}$ satisfies the following equation:

$$
\bar{\xi}_{i}(n)=L_{i} \bar{\xi}_{i}(n-1)-\lambda D\left(\sum_{k \neq i} \frac{b_{i k k}}{2} \bar{\theta}_{k}^{2}(n-1)-F_{i}(n-1)\right) .
$$

The error $F_{i}$ has the following estimate: For all $p$ with $1 \leq p \leq \infty$,

$$
\left\|F_{i}(n)\right\|_{l^{p}}=O\left(m_{i}^{2}\right)\left(1+n \nu_{i}\right)^{-3 / 2+1 / 2 p} \text { as } n \rightarrow \infty .
$$

Proof. The continuous function $\xi_{i}$ has the following estimate [3, 2]: For all $p$ with $1 \leq p \leq \infty$, for all $\alpha \geq 0$, and for $\sigma>0$ arbitrarily small,

$$
\text { (2.23) }\left(\int\left|\frac{\partial^{\alpha}}{\partial x^{\alpha}} \xi_{i}(x, t)\right|^{p} d x\right)^{1 / p}=O\left(m_{i}^{2}\right) O\left(\frac{\nu_{i}}{\lambda} t\right)^{-\alpha / 2-3 / 4+1 / 2 p+\sigma} \quad \text { as } t \rightarrow \infty \text {. }
$$

Then, (2.20) follows from (2.19) and (2.23). To show (2.22), we expand $\xi_{i}$ of (2.21) in a Taylor series about $\left(j-\lambda \lambda_{i}, n \lambda\right)$; we then use (2.17), (2.19), and (2.21) to obtain

$$
\begin{aligned}
& F_{i}(j+1, n-1) \\
&=-\sum_{k \neq i} \frac{b_{i k k}}{2}\left[2 \theta_{k}(j+1, n \lambda) \cdot K_{k}+K_{k}^{2}\right] \\
&-\lambda \int_{0}^{1}(1-s)\left(\sum_{k \neq i}\left(\frac{b_{i k k} b_{k k k}}{4} \theta_{k}^{3}-b_{i k k}\left(\frac{\nu_{k}}{4 \lambda}+\frac{\nu_{i}}{2 \lambda}\right) \theta_{k} \theta_{k_{x}}\right)\right. \\
&\left.+\left(\left(\frac{\nu_{i}}{2 \lambda}\right)^{2} \xi_{i_{x x}}\right)\right) \\
&+ \frac{\nu_{i}}{4 \lambda} \int_{0}^{1}(1-y)^{2}\left(\left(1+\lambda \lambda_{i}\right)^{2} \xi_{i_{x x}}\left(j+1+\lambda \lambda_{i}(y-1)+y, n \lambda\right)\right. \\
&\left.-\left(1-\lambda \lambda_{i}\right)^{2} \xi_{i_{x x}}\left(j+1+\lambda \lambda_{i}(y-1)-y, n \lambda\right)\right) d y .
\end{aligned}
$$


Here, $K_{k}$ is defined by (2.16). Then (2.22) follows from (2.14) and (2.23)(2.24).

\section{MAIN THEOREM}

We write $U_{i}=\bar{\theta}_{i}+\bar{\xi}_{i}+W_{i}$. From (2.7), (2.10), and (2.18)-(2.19) we have

$$
\sum_{j} \bar{\theta}_{i}(j, n)=\sum_{j} U_{i}(j, n)=m_{i}, \quad \sum_{j} \bar{\xi}_{i}(j, n)=0 \quad \forall n .
$$

This implies $\sum_{j} W_{i}(j, n)=0 \quad \forall n \geq 0$. Therefore, $W_{i}$ can be written as $D V_{i}$ with $V_{i}( \pm \infty, n)=0$, where

$$
V_{i}(j+1, n)=\sum_{k \leq j}\left(U_{i}(k, n)-\bar{\theta}_{i}(k, n)-\bar{\xi}_{i}(k, n)\right) .
$$

From (2.1), (2.12), and (2.21), $V_{i}$ satisfies

$$
\begin{aligned}
V_{i}(n)= & L_{i} V_{i}(n-1) \\
& +\lambda\left(E_{i}(n-1)+F_{i}(n-1)-N_{i}\left(\bar{\theta}_{i}(n-1), \bar{\xi}_{i}(n-1)+D V_{i}(n-1)\right)\right),
\end{aligned}
$$

where

$$
\begin{aligned}
N_{i}(a, b) & \equiv l_{i} \cdot\left(f(a+b)-f(0)-f^{\prime}(0)(a+b)\right)-\frac{1}{2} \sum_{k} b_{i k k} a_{k}^{2} \\
& =\sum_{j \neq k} \frac{b_{i j k}}{2} a_{j} a_{k}+\sum b_{i j k} a_{j} b_{k}+O\left(|a|^{3}+|b|^{2}\right),
\end{aligned}
$$

and $V=\sum V_{i} r_{i}, \bar{\theta}=\sum \bar{\theta}_{i} r_{i}, \bar{\xi}=\sum \bar{\xi}_{i} r_{i}$. Our goal is to show that $D V_{i}$ decays at the rate $O(1+n)^{-3 / 4+1 / 2 p+\sigma}$ in $l^{p}$, with $\sigma>0$ being arbitrarily small.

Theorem 3.1. Consider the system (1.1) with the initial condition (1.2) and with $\lambda$ satisfying (1.4). Let the discrete diffusion waves $\bar{\theta}_{i}(j, n), i=1, \ldots, N$, be defined by (2.10). Then there exists a constant $\varepsilon_{0}(\nu)>0$ such that if the initial data $U(0)$ satisfy

$$
\|U(0)\|_{l^{1}}+\|V(0)\|_{l^{1}} \leq \varepsilon_{0}
$$

where $V(0)$ is defined by (3.1), then the solutions of (1.1) and (1.2) converge to discrete diffusion waves. That is, for all $p$ with $1 \leq p \leq \infty$, and $\sigma>0$ arbitrarily small,

$$
\left\|U(n)-\sum_{i=1}^{N} \bar{\theta}_{i}(n) r_{i}\right\|_{l^{p}} \leq C(1+n \nu)^{-3 / 4+1 / 2 p+\sigma} \text { as } n \rightarrow \infty,
$$

for some positive constant $C$ independent of $n$.

Remark. From (2.11) and (3.5), we have that for all $1 \leq p \leq \infty,\|U(n)\|_{l^{p}}=$ $O(1+n \nu)^{-1 / 2+1 / 2 p}$ as $n \rightarrow \infty$. Thus, $U(n)$ decays in $l^{p}$ for $1<p \leq \infty$, but not in $l^{1}$. This decay rate is optimal because the estimate (2.11) is optimal. 
Proof of Theorem 3.1. Let $G_{i}(j, n)$ be the fundamental solution of the linear part of (3.2), i.e.,

$$
\begin{gathered}
G_{i}(n)=L_{i} G_{i}(n-1), \\
G_{i}(j, 0)= \begin{cases}1 & \text { if } j=0, \\
0 & \text { if } j \neq 0 .\end{cases}
\end{gathered}
$$

Then (3.2) can be converted into an "integral equation" through the help of $G_{i}$ as follows:

$$
\begin{aligned}
& V_{i}(j, n)= \sum_{k} G_{i}(j-k, n) V_{i}(k, 0) \\
&+\lambda \sum_{m=0}^{n-1} \sum_{k} G_{i}(j-k, n-m)\left(E_{i}(k, m)+F_{i}(k, m)-N_{i}(k, m)\right), \\
& i=1, \ldots, N .
\end{aligned}
$$

Here, $N_{i}(k, m)$ is an abbreviation for $N_{i}(\bar{\theta}, \bar{\xi}+D V)$ evaluated at $(k, m)$. We can also rewrite this formula in the following vector form:

$$
V(n)=G(n) * V(0)+\lambda \sum_{m=0}^{n-1} G(n-m) *(E(m)+F(m)-N(m)),
$$

where $G=\sum r_{i} G_{i} l_{i}$. Let us define a norm for $V$ : Given any arbitrarily small $\sigma>0$, let

$$
\|V\| \equiv|V|_{0, \infty}+|V|_{1,1}+|V|_{1, \infty}
$$

where

$$
\begin{gathered}
|V|_{0, \infty}=\sup _{n>0}(1+n \nu)^{1 / 4-\sigma}\|V(n)\|_{l^{\infty}}, \\
|V|_{1, p}=\sup _{n>0}(1+n \nu)^{3 / 4-1 / 2 p-\sigma}\|D V(n)\|_{l^{p}}, \quad 1 \leq p \leq \infty .
\end{gathered}
$$

Clearly, from (2.20) and (3.1) our theorem follows if we can show that the solutions of (3.8) satisfy $\|V\| \leq C$.

We need two lemmas.

Lemma 3.2. The fundamental solutions $G_{i}, i=1, \ldots, N$, have the following estimates: for $\alpha=0,1$, and all $p$ with $1 \leq p \leq \infty$,

$$
\left\|D^{\alpha} G_{i}(n)\right\|_{l^{p}} \leq C\left(1+n \nu_{i}\right)^{-\alpha / 2-1 / 2+1 / 2 p}, \quad i=1, \ldots, N .
$$

Proof. $G_{i}$ is the binomial distribution that has the following exact expression:

$$
G_{i}(j, n)=\left(\begin{array}{c}
n \\
(n+j) / 2
\end{array}\right) p^{(n+j) / 2} q^{(n-j) / 2}, \quad j+n=\text { even },
$$


where $p=\left(1+\lambda \lambda_{i}\right) / 2$ and $q=\left(1-\lambda \lambda_{i}\right) / 2$. Let $m, r$ be such that $m=n p+r$, $-q<r<p$. Further, let $j_{m}=2 m-n, k=(j-2 m+n) / 2$. Then

$$
\begin{gathered}
G_{i}(j, n)=G\left(j_{m}, n\right) \cdot \frac{(n-m) ! m !}{(m+k) !(n-m-k) !} p^{k} q^{-k} \\
D G_{i}(j, n+1)=\frac{r-k-q}{(n p+r+k+1) q} G_{i}(j, n) .
\end{gathered}
$$

By applying the Stirling formula when $n$ or $m+k$ or $n-m-k$ is large, we obtain that for large $n$ and $-n p+n \nu_{i} / 2 \leq k \leq n q-n \nu_{i} / 2$,

$$
\begin{gathered}
G_{i}(j, n) \sim \frac{1}{\sqrt{2 \pi n \nu_{i}}} \exp \left(-\frac{k^{2}}{n \nu_{i}}\right), \\
D G_{i}(j, n+1) \sim-\frac{k}{n \nu_{i}} \frac{1}{\sqrt{2 \pi n \nu_{i}}} \exp \left(-\frac{k^{2}}{n \nu_{i}}\right) .
\end{gathered}
$$

For $k$ outside the above domain, $G_{i}=O\left(e^{-\gamma n}\right)$ and $D G_{i}=O\left(e^{-\gamma n}\right)$ for some positive number $\gamma$. Then (3.12) follows from these expressions and the $L^{p}$ estimate of the heat kernel.

The next lemma follows easily.

Lemma 3.3. Let $\alpha, \beta, \gamma, \nu$ be positive constants. Then

(i) $\sum_{m=0}^{[n / 2]}(1+(n-m) \nu)^{-\beta}(1+m \nu)^{-\gamma}=O(1)(1+n \nu)^{-\alpha}$ if $\alpha \leq \beta, \alpha \leq$ $\beta+\gamma-1, \gamma \neq 1$, or if $\alpha<\beta, \alpha \leq \beta+\gamma-1, \gamma=1$;

(ii) $\sum_{m=[n / 2]+1}^{n}(1+(n-m) \nu)^{-\beta}(1+m \nu)^{-\gamma}=O(1)(1+n \nu)^{-\alpha}$ if $\alpha \leq \gamma$, $\alpha \leq \beta+\gamma-1, \beta \neq 1$, or if $\alpha<\gamma, \alpha \leq \beta+\gamma-1, \beta=1$;

(iii) $\quad \sum_{m=0}^{n}(1+(n-m) \nu)^{-\beta} e^{-\gamma m \nu}=O(1)(1+n \nu)^{-\alpha}$ if $\alpha \leq \beta$.

Now we use (3.8) to estimate $\|V(n)\|_{l^{\infty}},\|D V(n)\|_{l^{1}}$, and $\|D V(n)\|_{l^{\infty}}$ as follows:

$$
\begin{aligned}
\|V(n)\|_{l^{\infty}} \leq & \|G(n)\|_{l^{\infty}}\|V(0)\|_{l^{\infty}} \\
& +\lambda \sum_{m=0}^{n-1}\|G(n-m)\|_{l^{\infty}}\left(\|E(m)\|_{l^{1}}+\|F(m)\|_{l^{1}}+\|N(m)\|_{l^{1}}\right),
\end{aligned}
$$

$$
\|D V(n)\|_{l^{1}} \leq\|D G(n)\|_{l^{1}}\|V(0)\|_{l^{1}}
$$

$$
+\lambda \sum_{m=0}^{n-1}\|D G(n-m)\|_{l^{1}}\left(\|E(m)\|_{l^{1}}+\|F(m)\|_{l^{1}}+\|N(m)\|_{l^{1}}\right)
$$


$\|D V(n)\|_{l^{\infty}} \leq\|D G(n)\|_{l^{\infty}}\|V(0)\|_{l^{1}}$

$$
\begin{aligned}
+\lambda \sum_{k=0}^{[n / 2]}\|D G(n-m)\|_{l^{\infty}}\left(\|E(m)\|_{l^{1}}+\|F(m)\|_{l^{1}}+\|N(m)\|_{l^{1}}\right) \\
+\lambda \sum_{k=[n / 2]+1}^{n}\|D G(n-m)\|_{l^{1}}\left(\|E(m)\|_{l^{\infty}}+\|F(m)\|_{l^{\infty}}\right. \\
\left.+\|N(m)\|_{l^{\infty}}\right) .
\end{aligned}
$$

From (3.3), the nonlinear term $N$ has the following estimate:

$$
\begin{aligned}
\|N(\bar{\theta}, \bar{\xi}+D V)\|_{l^{p}}= & O(1)\left(\left\|\sum_{j \neq k} \bar{\theta}_{j} \bar{\theta}_{k}\right\|_{l^{p}}+\|\bar{\theta}\|_{l^{\infty}}^{2}\|\bar{\theta}\|_{l^{p}}\right) \\
& +O(1)\left(\|\bar{\theta}\|_{l^{\infty}}\|\bar{\xi}\|_{l^{p}}+\|\bar{\theta}\|_{l^{p}}\|\bar{\xi}\|_{l^{\infty}}+\|\bar{\xi}\|_{l^{\infty}}\|\bar{\xi}\|_{l^{p}}\right) \\
& +O(1)\left(\|\bar{\theta}\|_{l^{\infty}}+\|\bar{\xi}\|_{l^{\infty}}\right)\|D V\|_{l^{p}} \\
& +O(1)\left(\|\bar{\theta}\|_{l^{p}}+\|\bar{\xi}\|_{l^{p}}\right)\|D V\|_{l^{\infty}} \\
& +O(1)\|D V\|_{l^{\infty}}\|D V\|_{l^{p}}
\end{aligned}
$$

Let us call $\|U(0)\|_{l^{\prime}}$ by $\delta$. Then

$$
\left|m_{i}\right| \equiv\left|l_{i} \cdot \sum U(j, 0)\right| \leq \delta, \quad i=1, \ldots, N .
$$

From (2.9)-(2.10) and the fact that $\lambda_{j} \neq \lambda_{k}$, we have for all $p$ with $1 \leq p \leq \infty$,

$$
\left\|\bar{\theta}_{j}(n) \bar{\theta}_{k}(n)\right\|_{l^{p}}=O\left(\delta^{2} e^{-\beta \nu n}\right),
$$

for some $\beta$ with $0<\beta \leq \frac{1}{4} \min \left|\lambda_{j}-\lambda_{k}\right|$.

From (3.13), (3.16)-(3.17), (3.11), and Lemmas 2.1-2.2 and 3.2-3.3, we have

$$
\begin{aligned}
\|V(n)\|_{l^{\infty}} \leq & O(1+n \nu)^{-1 / 2}\|V(0)\|_{l^{1}} \\
+ & O(1) \sum_{m=0}^{n-1}(1+(n-m) \nu)^{-1 / 2} \\
& \cdot\left\{\left(\delta+\delta^{2}+\delta^{3}\right)(1+m \nu)^{-1}+\delta^{2} e^{-\beta m \nu}\right. \\
& +\delta^{3}(1+m \nu)^{-3 / 4+\sigma}+\delta^{4}(1+m \nu)^{-1+2 \sigma} \\
& +\left(\delta(1+m \nu)^{-3 / 4+\sigma}+\delta^{2}(1+m \nu)^{-1+2 \sigma}\right)\left(|V|_{1,1}+|V|_{1, \infty}\right) \\
& \left.+(1+m \nu)^{-1+2 \sigma}|V|_{1,1}|V|_{1, \infty}\right\} \\
\leq & O(1+n \nu)^{-1 / 4+\sigma}\|V(0)\|_{l^{1}}+(1+n \nu)^{-1 / 4+\sigma} \\
& \cdot\left(O(\delta)+O(\delta)\|V\|+\|V\|^{2}\right) .
\end{aligned}
$$


Similarly, we have

$$
\begin{aligned}
\|D V(n)\|_{l^{1}} \leq & O(1+n \nu)^{-1 / 4+\sigma}\|V(0)\|_{l^{1}} \\
& +(1+n \nu)^{-1 / 4+\sigma}\left(O(\delta)+O(\delta)\|V\|+\|V\|^{2}\right) .
\end{aligned}
$$

From (3.15)-(3.17) (3.11), and Lemmas 2.1-2.2 and 3.2-3.3, we have

$$
\begin{aligned}
\|D V(n)\|_{l^{\infty}} \leq & (1+n \nu)^{-1}\|V(0)\|_{l^{1}} \\
+ & O(1) \sum_{m=0}^{[n / 2]}(1+(n-m) \nu)^{-1} \\
& \cdot\left\{\left(\delta+\delta^{2}+\delta^{3}\right)(1+m \nu)^{-1}+\delta^{2} e^{-\beta m \nu}\right. \\
& +\delta^{3}(1+m \nu)^{-3 / 4+\sigma}+\delta^{4}(1+m \nu)^{-1+2 \sigma} \\
& +\left(\delta(1+m \nu)^{-3 / 4+\sigma}+\delta^{2}(1+m \nu)^{-1+2 \sigma}\right)\left(|V|_{1,1}+|V|_{1, \infty}\right) \\
+ & O(1) \sum_{m=[n / 2]+1}^{n}(1+(n-m) \nu)^{-1 / 2} \\
& \left\{\left(\delta+\delta^{2}+\delta^{3}\right)(1+m \nu)^{-3 / 2}+\delta^{2} e^{-\beta m \nu}\right. \\
& \left.+\delta^{3}(1+m \nu)^{-5 / 4+\sigma}+\delta^{4}(1+m \nu)_{1, \infty}\right\} \\
& +\left(\delta(1+m \nu)^{-5 / 4+\sigma}+\delta^{2}(1+m \nu)^{-3 / 2+2 \sigma}\right)|V|_{1, \infty} \\
& +(1+n \nu)^{-3 / 4+\sigma}\left(O(\delta)+O(\delta)\|V\|+\|V\|^{2}\right) . \\
\leq(1+n \nu)^{-3 / 4+\sigma}\|V(0)\| l^{1} & \left.+(1+m \nu)^{-3 / 2+2 \sigma}|V|_{1, \infty}^{2}\right\} \\
&
\end{aligned}
$$

We sum over these three inequalities and apply (3.9)-(3.11) to obtain

$$
\|V\| \leq C\left(\|V(0)\|_{l^{1}}+\delta+\delta\|V\|+\|V\|^{2}\right)
$$

for some positive constant $C$. Hence, if $\delta+\|V(0)\|_{l^{1}}$ is small, then $\|V\|$ is bounded. This completes the proof of Theorem 3.1.

\section{ACKNOWLEDGMENT}

This work was supported in part by the U.S. Department of Energy under grant DE-FG02-88ER25053 and in part by the Applied Mathematical Sciences subprogram of the Office of Energy Research, U.S. Department of Energy, under Contract W-31-109-Eng-38. The work was done when the author visited 
the Courant Institute of Mathematical Sciences, New York University. The author thanks Professor Tai-Ping Liu for helpful discussions.

\section{BIBLIOGRAPHY}

1. I.-L. Chern and T.-P. Liu, Convergence to diffusion waves of solutions for viscous conservation laws, Comm. Math. Phys. 110 (1987), 503-517.

2. __ Erratum, convergence of diffusion waves of solutions for viscous conservation laws, Comm. Math. Phys. 120 (1989), 525-527.

3. I.-L. Chern, Multiple-mode diffusion waves for viscous nonstrictly hyperbolic conservation laws, preprint, MCS-P134-0290, Math. Comp. Div., Argonnc .vational Lab., 1990.

4. E. Hopf, The partial differential equation $u_{t}+u u_{x}=\mu u_{x x}$, Comm. Pure Appl. Math. 3 (1950), 201-230.

5. S. Kawashima, Systems of a hyperbolic-parabolic composite type, with applications to the equations of magnetohydrodynamics, Doctoral thesis, Kyoto University, 1983.

6. __ Large-time behavior of solutions to hyperbolic-parabolic systems of conservation laws and applications, Proc. Roy. Soc. Edinburgh Sect. A 106 (1987), 169-194.

7. T.-P. Liu, Pointwise convergence to $N$-waves for solutions of hyperbolic conservation laws, Bull. Inst. Math. Acad. Sinica 15 (1987), 1-17.

8. A. Matzumura and T. Nishida, The initial value problem for the equations of motion of viscous and heat-conductive gases, J. Math. Kyoto Univ. 20 (1980), 67-104

Mathematics and Computer Science Division, Argonne National laboratory, ArGONNE, ILLINOIS 60439

Department of Mathematics, The University of Chicago, Chicago, Illinois 60637

E-mail address: chern@mcs.anl.gov 